\title{
Monte Carlo simulation of electron transport in degenerate and inhomogeneous semiconductors
}

Mona Zebarjadi, Ceyhun Bulutay, Keivan Esfarjani, and Ali Shakouri

Citation: Appl. Phys. Lett. 90, 092111 (2007); doi: 10.1063/1.2709999

View online: http://dx.doi.org/10.1063/1.2709999

View Table of Contents: http://aip.scitation.org/toc/apl/90/9

Published by the American Institute of Physics

\section{Articles you may be interested in}

Improved Monte Carlo method for the study of electron transport in degenerate semiconductors Journal of Applied Physics 84, 3706 (1998); 10.1063/1.368547

Tunneling-assisted Poole-Frenkel conduction mechanism in $\mathrm{HfO} \mathrm{O}_{2}$ thin films Journal of Applied Physics 98, 113701 (2005); 10.1063/1.2135895

Monte-Carlo-based spectral gain analysis for terahertz quantum cascade lasers Journal of Applied Physics 105, 123102 (2009); 10.1063/1.3147943

Modeling of electron-electron scattering in Monte Carlo simulation of quantum cascade lasers Journal of Applied Physics 97, 043702 (2005); 10.1063/1.1840100

The Three-Dimensional Poole-Frenkel Effect Journal of Applied Physics 39, 4871 (2003); 10.1063/1.1655871

The Poole-Frenkel Effect with Compensation Present Journal of Applied Physics 39, 5600 (2003); 10.1063/1.1656022

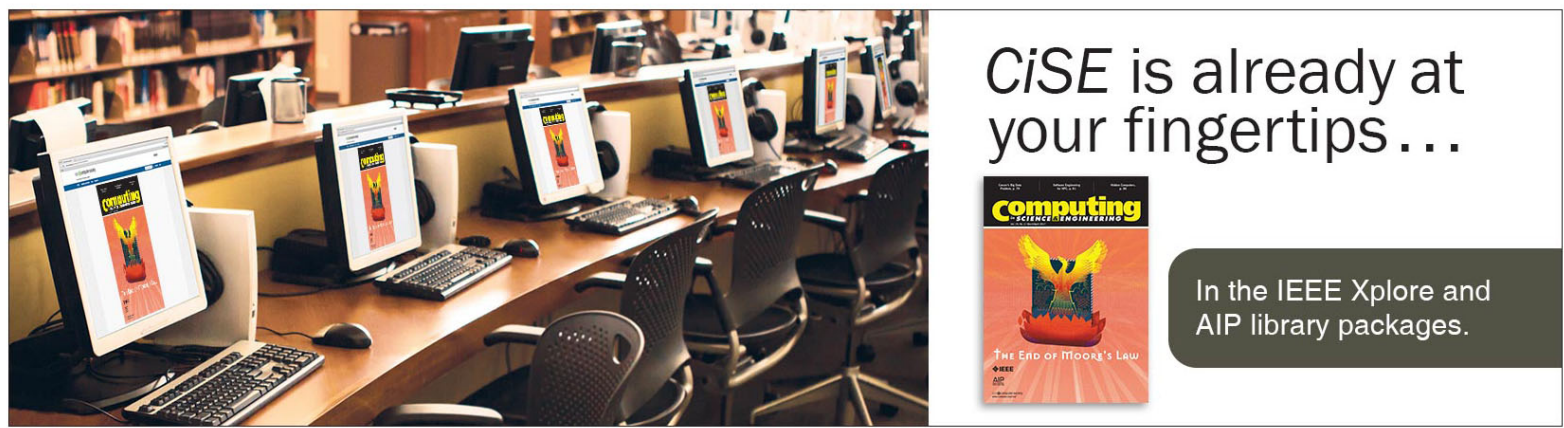




\title{
Monte Carlo simulation of electron transport in degenerate and inhomogeneous semiconductors
}

\author{
Mona Zebarjadia) \\ Department of Electrical Engineering, University of California, Santa Cruz, California 95064 \\ Ceyhun Bulutay \\ Department of Physics, Bilkent University, Ankara 06800, Turkey \\ Keivan Esfarjani \\ Department of Physics, University of California, Santa Cruz, California 95064 \\ Ali Shakouri \\ Department of Electrical Engineering, University of California, Santa Cruz, California 95064
}

(Received 14 November 2006; accepted 26 January 2007; published online 28 February 2007)

\begin{abstract}
An algorithm is proposed to include Pauli exclusion principle in Monte Carlo simulations. This algorithm has significant advantages to implement in terms of simplicity, speed, and memory storage; therefore it is ideal for the three-dimensional device simulators. The authors show that even in moderately high applied fields, one can obtain the correct electronic distribution. They give the correct definition for electronic temperature and show that in high applied fields, the quasi-Fermi level and electronic temperature become valley dependent. The effect of including Pauli exclusion principle on the band profile, electronic temperature, and quasi-Fermi level for the inhomogeneous case of a single barrier heterostructure is illustrated. (C) 2007 American Institute of Physics.
\end{abstract}

[DOI: $10.1063 / 1.2709999$ ]

Many of today's interesting microelectronic devices are working in high doping concentrations up to $10^{20} \mathrm{~cm}^{-3}$. Degenerate semiconductors are important for thermoelectric and thermionic energy conversion devices and they are also used in the highly doped source/drain regions of advanced transistors. At high carrier densities, the electron distribution is highly affected by its fermionic nature. Therefore, Pauli exclusion principle (PEP) needs to be included in the theoretical analysis of electron transport in degenerate semiconductor-based structures.

If the electron wavelength is smaller than the characteristic lengths present in the structure, the Boltzmann transport equation (BTE) can be used as an appropriate governing equation for the device. The factor $(1-f)$ in BTE, which indicates the probability of the final state to be unoccupied, is present in the scattering term as a result of PEP.

$$
\begin{aligned}
\frac{\partial f(\mathbf{r}, \mathbf{k}, t)}{\partial t}= & -\mathbf{v} \cdot \boldsymbol{\nabla} f(\mathbf{r}, \mathbf{k}, t)-\mathbf{F} / \hbar \cdot \nabla_{k} f(\mathbf{r}, \mathbf{k}, t) \\
& +\sum_{k^{\prime}}\left\{W\left(\mathbf{k}^{\prime}, \mathbf{k}\right) f\left(\mathbf{r}, \mathbf{k}^{\prime}, t\right)[1-f(\mathbf{r}, \mathbf{k}, t)]\right. \\
& \left.-W\left(\mathbf{k}, \mathbf{k}^{\prime}\right) f(\mathbf{r}, \mathbf{k}, t)\left[1-f\left(\mathbf{r}, \mathbf{k}^{\prime}, t\right)\right]\right\} .
\end{aligned}
$$

Here, $f$ is the nonequilibrium distribution function, $\mathbf{F}$ is the applied electric force, $\mathbf{v}$ is the group velocity, and $W$ the scattering rate. The ensemble Monte Carlo (MC) simulation is accepted as a powerful numerical technique to solve BTE and is widely used to simulate transport in semiconductor devices. There have been some attempts to include PEP in MC simulations of degenerate semiconductors. All of these attempts have been based on the rejection method. At each scattering step, scattering to the final state is accepted with

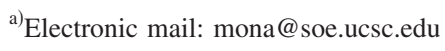

the probability of 1- $f$. However, in a MC simulation $f$ is not a priori known.

In a single-electron MC simulation Bosi and Jacoboni ${ }^{1}$ suggested using $f(\mathbf{k}, t)$ obtained from the simulation itself up to the time $t$ at which the scattering is attempted. They evaluate $f(\mathbf{k}, t)$ on a grid in $k$-space. Lugli and Ferry ${ }^{2}$ (LF) proposed using the same method in ensemble MC simulation, but substituting the averaging over time by ensemble average at each time step. This algorithm is working well at high fields but it is not suitable for low fields and highly degenerate cases. In low fields, it is reported that the algorithm leads to some unphysical results such as the decrease of average electron energy with the increase of electric field and values of electron distribution function exceeding $1 .^{3}$ In order to improve the LF method, Borowik and Thobel ${ }^{3}$ and Borowik and Adamowicz ${ }^{4}$ suggested adding scattering-out terms into the simulation by introducing virtual scatterings in order to avoid $f>1$. With this method they were able to rebuild the Fermi-Dirac distribution with a small deviation. We believe that in principle LF method is free from these artifacts at the cost of excessive $k$-space grid points, also implying a large number of simulated particles. Besides, if PEP is also checked after free flights, the distribution function should not exceed $1 .^{5}$ Another inconvenience of the LF method is that this algorithm is suitable for a homogeneous steady state situation. In transient simulations and in inhomogeneous devices, tabulating the distribution function at various locations and times can require an unmanageable computation time and storage.

Fischetti and Laux ${ }^{6}$ proposed to overcome this difficulty by approximating the local electron distribution by a quasiequilibrium Fermi-Dirac distribution with the following definition for local electronic temperature: 


$$
\frac{3}{2} k_{B} T_{\mathrm{el}}(r)=\left\{\begin{array}{cc}
\langle E(r)\rangle & \text { nondegenerate } \\
\mu(r) & \text { degenerate, }
\end{array}\right.
$$

where $\mu(r)$ is the local quasi-Fermi level and $\langle E(r)\rangle$ is the local average kinetic energy of electrons. The above definition for electronic temperature is not correct at high concentrations when the Maxwell-Boltzmann distribution is not valid. The correct definition of electronic temperature should converge to the lattice temperature at zero electric field and should exceed it under an applied field. Moreover, under the applied bias, translational energy should be subtracted, because temperature is defined by fluctuations of electron velocities around its mean drift value. These considerations lead us to the following definitions for the distribution function and electronic temperature of each valley:

$$
\begin{aligned}
& f_{v}\left(E, \mu_{v}, T_{\mathrm{el}}^{v}\right) \\
& \quad=\frac{1}{\exp \left(\left[E_{v}\left(\left|\mathbf{k}-\mathbf{k}_{d}^{v}(\mathbf{r})\right|\right)-\mu_{v}(\mathbf{r})\right] / k_{B} T_{\mathrm{el}}^{v}(\mathbf{r})\right)+1}, \\
& T_{\mathrm{el}}^{v}(\mathbf{r})=\frac{2}{3 k_{B}}\left(\left\langle E_{v}\left(\mathbf{k}-\mathbf{k}_{d}^{v}(\mathbf{r})\right)\right\rangle-\left\langle E_{v}(\mathbf{r})\right\rangle_{0}\right)+T_{\text {lattice }} .
\end{aligned}
$$

Here $v$ is the valley index, and $\left\langle E_{v}(\mathbf{r})\right\rangle_{0}$ is the local average energy of electrons in equilibrium at zero electric field and is calculated analytically at each time step [see Eq. (5)]. $\mathbf{k}_{d}^{v}(\mathbf{r})$ is the local drift wave vector, which is the average wave vector of all the particles at position $\mathbf{r}$ and in valley $v$. For moderately high field electrons in different valleys are not in equilibrium with each other, so separate Fermi spheres with different chemical potentials and temperatures need to be defined for each valley. Therefore, all quantities are valley dependent. Also in inhomogeneous cases such as heterostructures, we need to discretize the $x$ space and define the Fermi level and the electronic temperature locally.

The implementation of the algorithm in the MC simulation is straightforward. At each time step, the drift wave vector $\left(\mathbf{k}_{d}\right)$, average energy of electrons $\left(\left\langle E\left(\mathbf{k}-\mathbf{k}_{d}\right)\right\rangle\right)$, and local charge $\left(n_{c}^{v}\right)$ are calculated. Then electronic temperature, Fermi level, and $\left\langle E_{v}\right\rangle_{0}$ for each valley can be calculated, using Eqs. (3)-(5), respectively.

$$
\begin{aligned}
& n_{c}^{v}=\int_{-\infty}^{\infty} f\left(\varepsilon, \mu_{v}, T_{\mathrm{el}}^{v}\right) g_{v}(\varepsilon) d \varepsilon, \\
& \left\langle E_{v}(\mathbf{r})\right\rangle_{0}=\int_{-\infty}^{\infty}\left(\varepsilon-\varepsilon_{c}^{v}\right) f\left(\varepsilon, \mu_{v}(\mathbf{r}), T_{\text {lattice }}\right) g_{v}(\varepsilon) d \varepsilon,
\end{aligned}
$$

where $g_{v}(\varepsilon)$ is the density of states and $\varepsilon_{c}^{v}$ is the bottom of the conduction band in the valley $\nu$. These updated quantities are used in Eq. (2) for scattering probabilities into final states at the next iteration.

Figure 1 shows the distribution function at zero electric field obtained from the present algorithm in comparison with analytical Fermi-Dirac (FD) distribution. The deviation is negligible in most of the energy range. However, using definitions of Ref. 6 [Eq. (1)], the obtained final distribution is totally different from the FD distribution. It also shows the results of implementing the algorithm with a low applied field. As expected some of the electrons below Fermi level are pushed to higher energy levels producing thereby heating.

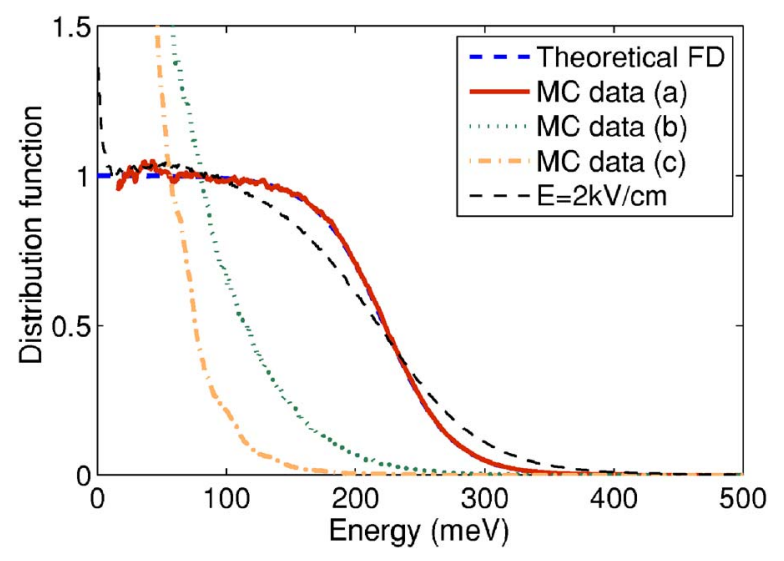

FIG. 1. (Color online) Electron energy distribution function at equilibrium for GaAs at room temperature and the doping of $10^{19} \mathrm{~cm}^{-3}$. Theoretical Fermi-Dirac function is plotted for comparison. The rest are Monte Carlo simulation results implementing (a) present algorithm, (b) algorithm of Ref. 6 [Eq. (1)], and (c) without Pauli exclusion principle. The last data are the result of present algorithm for low applied electric field of $2 \mathrm{kV} / \mathrm{cm}$.

Figure 2 shows a comparison between our algorithm and experimental results. ${ }^{7}$ The experiment has been done at $77 \mathrm{~K}$ on Te-doped GaAs. In low temperatures, impurity scattering is the dominant scattering mechanism. In this simulation, we have included both ionized and neutral impurity scatterings. Neutral impurities are considered as hard spheres with the potential of $35 \mathrm{eV}$ and radius of $2 \AA$. A binding energy of $0.03 \mathrm{eV}$ is considered for tellurium in GaAs. ${ }^{8}$ Polar optical and acoustic phonons are also included, both as inelastic processes. Although there is some deviation compared to experimental results, our results are closer to the experiment compared to the previous work of Ref. 1.

In Fig. 3 we compare the distribution function obtained from our suggested algorithm with that of the LF method. Scattering rates and band structure are the same for both cases and the only difference is the implementation of PEP. The agreement between the present method and the LF method suggests that the present algorithm works very well even at moderately high fields of the order of several $10 \mathrm{kV} / \mathrm{cm}$. Our modeling (nonparabolic multivalley band structure) becomes questionable at such fields for GaAs, so this should only be considered as a confirmation of the va-

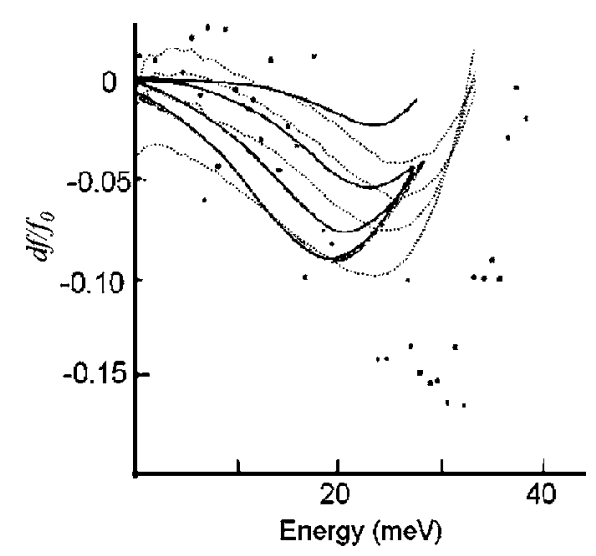

FIG. 2. Relative change of the electronic distribution to the equilibrium (FD distribution) at $77 \mathrm{~K}$ temperature and the doping density of $5 \times 10^{17} \mathrm{~cm}^{-3}$. Experimental results (solid curve) are after Ref. 8. MC data obtained from our simulation for the same applied electric fields $(200,400,600$, and $900 \mathrm{~V} / \mathrm{cm}$ from top to bottom) are shown by dotted lines. Previous MC simulation results of Ref. 1 are shown by dots (at $900 \mathrm{~V} / \mathrm{cm}$ ). 


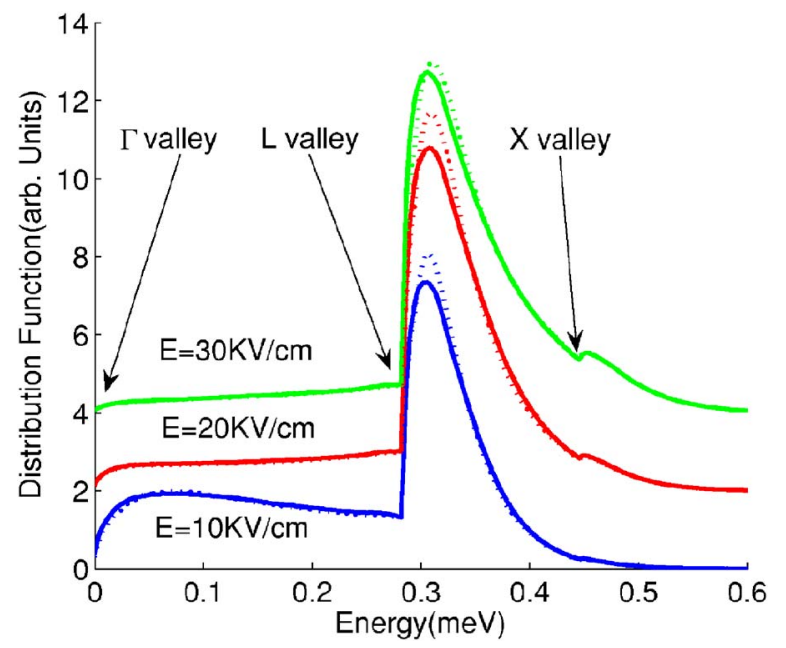

FIG. 3. (Color online) Comparison between LF method and the present method: distribution function for three different applied electric fields. Solid lines are obtained from the present algorithm and dots are obtained from LF method. For more clarity, the three graphs are shifted upwards by two units, and different valley minima are shown with an arrow.

lidity of our method and not a quantitative prediction of the electronic distribution at high fields. The figure is plotted for high doping of $10^{19} \mathrm{~cm}^{-3}$. In such a high doping, the LF method gives reasonable results with at least 700000 electrons (this large number of electrons is essential for reproducing the correct results especially at low fields); however, the present method is working well even with a sample of 10000 electrons implying a reduction in CPU time and memory by a factor of 70 ! Moreover, even with the same number of electrons the LF method uses 4.7 times the memory and it is $15 \%$ slower in comparison with the present method. These simulations were done on a personal computer with Pentium(R) 4 processor and 2.0 Gbytes of memory. It is noticeable that the agreement with the LF method, at high fields, is obtainable only if the quasi-Fermi level is defined separately for each valley.

Finally, we applied this approach to the inhomogeneous case of a single barrier heterostructure. In the absence of bias (equilibrium case) the quasi-Fermi level is constant throughout the whole device. Without considering PEP, however, the distribution would not lead to a constant Fermi level. Figure 4 shows the electronic temperature and quasi-Fermi level under a low applied bias, with and without applying PEP. The simulation is at room temperature. Without PEP, electrons are much colder than the lattice temperature especially in the contact layers where Fermi level is within the conduction band. In this case electrons would go from the barrier to the lower occupied states in the contacts and overpopulate them. This would lead to an artificial band bending which is shown in Fig. 4. By including degeneracy in the calculation, distribution leads to a continuous Fermi level and the electronic temperature is close to the lattice temperature. Cooling of electrons before the barrier and heating of them after the barrier can be explained as Peltier cooling and heating, and heating inside the barrier is a result of Joule heating. The transition between nonlinear thermionic emission cooling

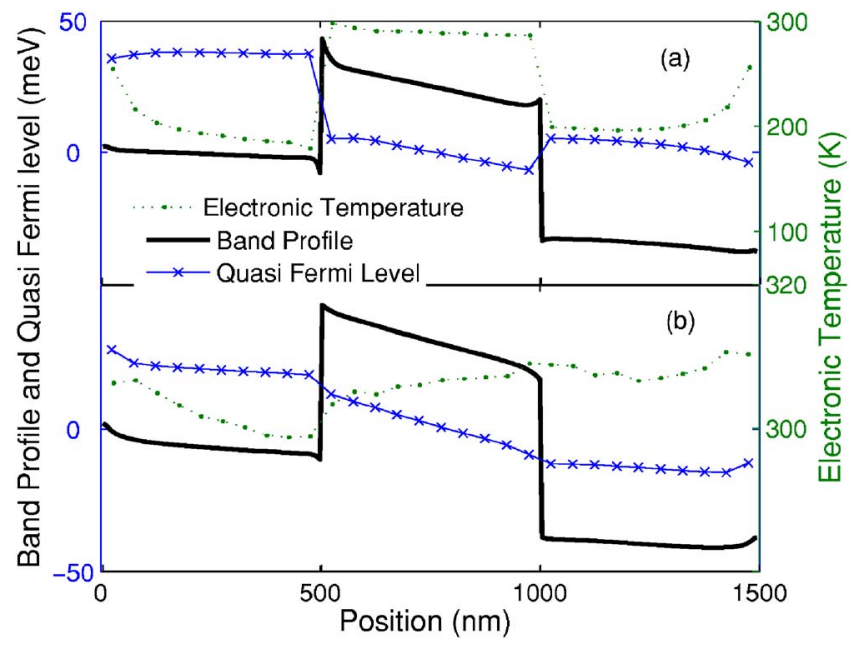

FIG. 4. (Color online) Band profile, quasi-Fermi level, and electronic temperature for InGaAs/InGaAsP/InGaAs heterostructure at room temperature under a low applied bias (a) without and (b) with PEP. Note that in (b) they are continuous across the junctions.

and linear transport is discussed in a recent publication. ${ }^{9} \mathrm{We}$ need to add that the irregularities at both ends of the sample stem from the injection mechanism imposed at the boundaries. They would become irrelevant for wider contact regions.

In summary, we proposed a theory which describes with good accuracy transport in degenerate and inhomogeneous semiconductors. It was shown that the algorithm requires much less time and memory storage compared to LF and similar methods. This allows the treatment of inhomogeneous systems, which is almost an impossible task with the LF method; therefore it is ideal for the three-dimensional device simulators. We have also given a definition for the electronic temperature, which leads to the correct distribution function in good agreement with these methods. Comparisons with analytical results at zero bias and with other algorithms and also experimental data under applied bias were also presented. Furthermore, the effect of including PEP in a heterostructure on the band profile, electronic temperature, and the quasi-Fermi level was illustrated. The theory correctly predicts a continuous electronic temperature profile and quasi-Fermi level across the junctions.

One of the authors (M.Z.) is thankful to U. Ravaioli for helpful discussions. The authors also wish to acknowledge the support by ONR Thermionic Energy Conversion Center MURI.

${ }^{1}$ S. Bosi and C. Jacoboni, J. Phys. C 9, 315 (1976).

${ }^{2}$ P. Lugli and D. K. Ferry, IEEE Trans. Electron Devices 32, 2431 (1985).

${ }^{3}$ P. Borowik and J. L. Thobel, J. Appl. Phys. 84, 3706 (1998).

${ }^{4}$ P. Borowik and L. Adamowicz, Physica B 365, 235 (2005).

${ }^{5}$ P. Tadyszak, F. Danneville, A. Cappy, L. Reggiani, L. Varani, and L. Rota, Appl. Phys. Lett. 69, 1450 (1996).

${ }^{6}$ M. V. Fischetti and S. E. Laux, Phys. Rev. B 38, 9721 (1988).

${ }^{7}$ W. Jantsch and H. Bruker, Phys. Rev. B 15, 4014 (1977).

${ }^{8}$ S. M. Sze, Semiconductor Devices, Physics and Technology (Wiley, New York, 1985).

${ }^{9}$ M. Zebarjadi, A. Shakouri, and K. Esfarjani, Phys. Rev. B 74, 195331 (2006). 